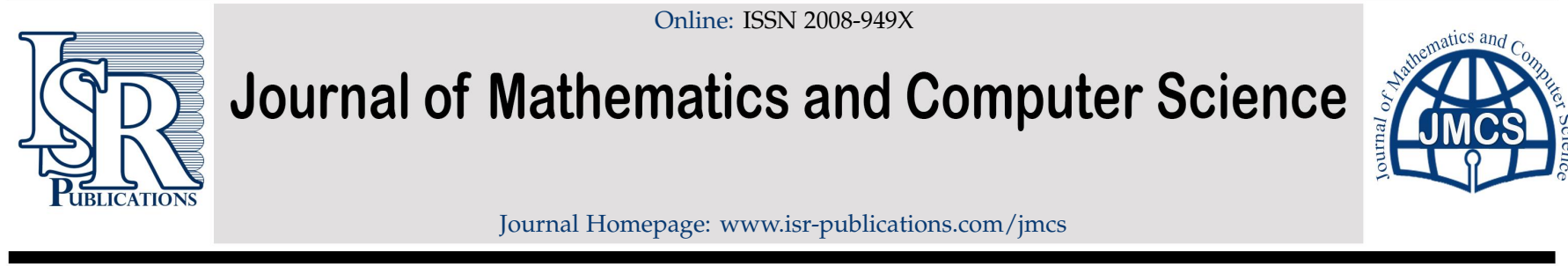

\title{
Generalized e-closed sets and generalized e-continuity in in- tuitionistic fuzzy topology
}

\author{
S. S. Thakur ${ }^{\mathrm{a}, *}$, Chandra Prakash Rathor ${ }^{\mathrm{b}}$, Mahima Thakur ${ }^{\mathrm{a}}$ \\ ${ }^{a}$ Department of Applied Mathematics, Jabalpur Engineering College, Jabalpur (M.P.) 482011, India. \\ ${ }^{b}$ Department of Mathematics, Rani Durgavati University, Jabalpur (M.P.) 482001, India.
}

\begin{abstract}
The purpose of this paper is to introduce and study the concepts of intuitionistic fuzzy generalized e-closed sets and intuitionistic fuzzy generalized e-open sets in intuitionistic Fuzzy topological space. We investigate some of their properties. Further the notion of intuitionistic fuzzy e $\mathrm{T}_{1 / 2}$ spaces, intuitionistic fuzzy GEO-connectedness, intuitionistic fuzzy GEO-compactness are introduced and studied.
\end{abstract}

Keywords: Intuitionistic fuzzy sets, intuitionistic fuzzy topology, intuitionistic fuzzy ge-closed sets and intuitionistic fuzzy ge-continuity.

2020 MSC: 54A40, 03F55.

(C)2022 All rights reserved.

\section{Introduction}

The concept of fuzzy sets was introduced by Zadeh [36]. Using the concept of fuzzy set Chang [4] introduced the fuzzy topological spaces. In 1970 Levine [12] introduced the concept of generalized closed sets in topology. He generalized some of well known results of general topology replacing closed set by generalized closed sets. Levine [12] also introduced the concept of generalized open sets and a new type separation axiom called $\mathrm{T}_{1 / 2}$-Axiom, using generalized closed sets. In 1991 Balchandran et al. [2] used the concepts of generalized closed and generalized open set to introduce and study of concept of generalized open connectedness and generalized open compactness in topology. In the same paper Balchandran et al. [2] introduced the concepts of generalized continuous and generalized closed irresolute mappings. After the publication of Balchandran et al. [2] paper many authors such as Dunham and Levine [7], Hatir and Erdogan [10], Maki and coworkers [17, 18] studied various weak and strong forms of generalized closed set and generalized continuity in topology.

In 1995 Thakur and Malviya [35] extended the concept of generalized closed sets in fuzzy topology. In the same year they extended the concepts of fuzzy generalized continuity [19] and fuzzy generalized closed irresoluteness of mappings [34] in fuzzy topology. In the recent years a large number of Authors

\footnotetext{
*Corresponding author

Email address: samajh_singh@rediffmail.com (S. S. Thakur)

doi: $10.22436 /$ jmcs.025.03.02
}

Received: 2021-02-20 Revised: 2021-04-27 Accepted: 2021-05-17 
such as Chan [11], Thakur and Khare [32,33] studied various weak and strong forms of generalized closed sets and generalized continuity in fuzzy topology.

In 1997, Coker [5] introduced the concepts of intuitionistic fuzzy topological spaces as a generalization of fuzzy topological spaces. After the introduction of intuitionistic fuzzy topology by Coker $[5,6,8]$ and many mathematicians such as Coker and coworkers [13-16, 31] have been extended various fuzzy topological concepts in intuitionistic fuzzy topology. Thakur and Chaturvedi $[25,29,30]$ were the first contributors who considered generalized closed sets and generalized continuity in intuitionistic fuzzy topology. In the same time period, they [26-28] also extended the concept of fuzzy rg-closed sets and fuzzy rg-continuity in intuitionistic fuzzy topology. Further, Thakur and Bajpai [20-24] studied strong and weak forms of intuitionistic fuzzy g-closed sets and intuitionistic fuzzy g-continuity.

In the present paper, we extend the concept of ge-closed sets in intuitionistic fuzzy topology and study some of the basic properties regarding it. We also introduce the intuitionistic fuzzy GEO-connected, intuitionistic fuzzy GEO-compact and intuitionistic fuzzy $\mathrm{eT}_{1 / 2}$ spaces and obtained some characterizations and several preservation theorems of such spaces.

\section{Preliminaries}

This section contains some basic definitions and preliminary results which will be needed in the sequel. First we present the fundamental definitions obtained by Atanassov and Coker.

Definition 2.1 ([1]). Let $X$ be a nonempty fixed set. An intuitionistic fuzzy set $A$ is an object having the form $A=\left\{\left\langle x, \mu_{A}(x), v_{A}(x)>: x \in X\right\}\right.$, where the functions $\mu_{A}: X \rightarrow I$ and $v_{A}: X \rightarrow$ I denote the degree of membership namely $\mu_{A}(x)$ and the degree of nonmembership (namely $\nu_{A}(x)$ ) of each element $x \in X$ to the set $A$, respectively, and $0 \leqslant \mu_{A}(x)+v_{A}(x) \leqslant 1$ for each $x \in X$.

Obviously, every fuzzy set $A$ on a nonempty set $X$ is an intuitionistic fuzzy set having the form $A=\left\{<x, \mu_{A}(x), 1-\mu_{A}(x)>: x \in X\right\}$.

For the basic properties of intuitionistic fuzzy set the researchers should refer [1].

Definition 2.2 ([5]). An intuitionistic fuzzy topology on a nonempty set $X$ is a family $\tau$ of intuitionistic fuzzy sets in $X$ satisfy the following axioms:

(a) $\tilde{0}, \tilde{1} \in \tau$;

(b) $\mathrm{G}_{1} \cap \mathrm{G}_{2} \in \tau$ for any $\mathrm{G}_{1}, \mathrm{G}_{2} \in \tau$;

(c) $\cup G_{i} \in \tau$ for any arbitrary family $\left\{G_{i}: i \in j\right\} \subseteq \tau$.

In this case the pair $(X, \tau)$ is called an intuitionistic fuzzy topological space and each intuitionistic fuzzy set in $\tau$ is known as an intuitionistic fuzzy open set in $X$.

Definition 2.3 ([5]). The Complement of $A^{c}$ of an intuitionistic fuzzy open set $A$ is an intuitionistic fuzzy topological space $(X, \tau)$ is called an intuitionistic fuzzy closed set in $X$.

Definition 2.4 ([5]). Let $(X, \tau)$ be an intuitionistic fuzzy topological space and let $A=\left\langle x, \mu_{A}(x), v_{A}(x)>\right.$ be an intuitionistic fuzzy set in $X$. Then the intuitionistic fuzzy interior and intuitionistic fuzzy closure of A are defined by

$$
\begin{aligned}
& \operatorname{int}(A)=\cup\{G \mid G \text { is an intuitionistic fuzzy open set in } X \text { and } G \subseteq A\}, \\
& \operatorname{cl}((A)=\cap\{K \mid K \text { is an intuitionistic fuzzy closed set in } X \text { and } A \subseteq K\} .
\end{aligned}
$$

Definition 2.5 ([14]). Let $c(\alpha, \beta)$ be an intuitionistic fuzzy point in $X$ and $A=<X, \mu_{A}, \gamma_{A}>$ is an intuitionistic fuzzy set in $X$. Then $c(\alpha, \beta) \subseteq A$ if and only if $c_{\alpha} \subseteq \mu_{A}$ and $1-c_{1-\beta} \geqslant \gamma_{A}$, or equivalently, $\alpha \subseteq \mu_{A}(c)$ and $\beta \supseteq \gamma_{A}(c)$. 
Definition 2.6 ([30]). Let $(X, \tau)$ and $(Y, \sigma)$ be two intuitionistic fuzzy topological space and $f: X \rightarrow Y$ be a function. Then $f$ is said to be intuitionistic fuzzy continuous if the preimage of each intuitionistic fuzzy open set in $\mathrm{Y}$ is an intuitionistic fuzzy open set in $\mathrm{X}$.

Definition 2.7 ([30]). A family $\left\{A_{i}: i \in \Lambda\right\}$ of intuitionistic fuzzy sets in $X$ is said to be intuitionistic fuzzy open cover of $X$ if $\cup\left\{A_{i}: i \in \Lambda\right\}=\tilde{1}$ and a finite subfamily of an intuitionistic fuzzy open cover of $X$ which also an intuitionistic fuzzy open cover of $X$ is called a finite subcover $\left\{A_{i}: i \in \Lambda\right\}$.

Definition 2.8 ([30]). An intuitionistic fuzzy topological space $(X, \tau)$ is called intuitionistic fuzzy compact if every intuitionistic fuzzy open cover of $X$ has a finite subcover.

Definition 2.9 ([30]).

(a) An intuitionistic fuzzy topological space $(X, \tau)$ is called intuitionistic fuzzy $C_{5}$-disconnected, if there exists fuzzy weakly separated non-zero intuitionistic fuzzy sets $A$ and $B$ in $(X, \tau)$ such that $A \cup B=\tilde{1}$.

(b) $(X, \tau)$ is called intuitionistic fuzzy $C_{5}$-connected if $(X, \tau)$ is not intuitionistic fuzzy $C_{5}$-disconnected.

Definition 2.10 ([3]). An intuitionistic fuzzy set $A$ of an intuitionistic fuzzy topological space $(X, \tau)$ is called intuitionistic fuzzy regular open set (resp. intuitionistic fuzzy regular closed set) if $A=\operatorname{int}(\operatorname{cl}(A))$ (resp. $A=\operatorname{cl}(\operatorname{int}(A))$.

Definition 2.11 ([3]). The $\delta$-interior of an intuitionistic fuzzy set $A$ of an intuitionistic fuzzy topological space $(X, \tau)$ is the union of all intuitionistic fuzzy regular open sets contained in $A$.

Definition 2.12 ([3]). An intuitionistic fuzzy set $A$ of an intuitionistic fuzzy topological space $(X, \tau)$ is called intuitionistic fuzzy $\delta$-open if $A=\operatorname{\delta int}(A)$. The complement of intuitionistic fuzzy $\delta$-open set is called intuitionistic fuzzy $\delta$-closed.

Lemma 2.13. An intuitionistic fuzzy set $A$ is an intuitionistic fuzzy $\delta$-closed if and only if $A=\delta \mathrm{cl}(A)$.

Definition 2.14. An intuitionistic fuzzy set $A$ of an intuitionistic fuzzy topological space $(X, \tau)$ is called:

(a) intuitionistic fuzzy semiopen if $A \subseteq \mathrm{cl}(\operatorname{int}(A))[8]$;

(b) intuitionistic fuzzy $\alpha$-open if $A \subseteq \operatorname{int}(\operatorname{cl}(\operatorname{int}(A)))$ [8];

(c) intuitionistic fuzzy $\beta$-open if $A \subseteq \operatorname{cl}(\operatorname{int}(\mathrm{cl}(A)))$ [8];

(d) intuitionistic fuzzy preopen if $A \subseteq \operatorname{int}(\operatorname{cl}(A))$ [8];

(e) intuitionistic fuzzy $\gamma$-open if $A \subseteq \operatorname{cl}(\operatorname{int}(A)) \cup \operatorname{int}(\operatorname{cl}(A))$ [9];

(f) intuitionistic fuzzy $\delta$-preopen if $A \subseteq \operatorname{int}(\delta \operatorname{cl}(A))[3]$;

(g) intuitionistic fuzzy $\delta$-semiopen if $A \subseteq \operatorname{cl}(\operatorname{sint}(A))[3]$.

The complements of an intuitionistic fuzzy semiopen (resp. intuitionistic fuzzy $\alpha$-open, intuitionistic fuzzy $\beta$-open, intuitionistic fuzzy preopen, intuitionistic fuzzy $\gamma$-open, intuitionistic fuzzy $\delta$-preopen, intuitionistic fuzzy $\delta$-semiopen) is called intuitionistic fuzzy semiclosed (resp. intuitionistic fuzzy $\alpha$-closed, intuitionistic fuzzy $\beta$-closed, intuitionistic fuzzy preclosed, intuitionistic fuzzy $\gamma$-closed, intuitionistic fuzzy $\delta$-preclosed, intuitionistic fuzzy $\delta$-semiclosed).

Definition 2.15 ([3]). An intuitionistic fuzzy set $A$ of an intuitionistic fuzzy topological space $(X, \tau)$ is called:

(a) intuitionistic fuzzy e-open if $A \subseteq \operatorname{cl}(\operatorname{\delta int}(A)) \cup \operatorname{int}(\delta \mathrm{cl}(A))$;

(b) intuitionistic fuzzy e-closed if $A \supseteq \operatorname{cl}(\operatorname{sint}(A)) \cap \operatorname{int}(\delta \operatorname{cl}(A))$.

Lemma 2.16. An intuitionistic fuzzy set A in intuitionistic fuzzy topological space $(X, \tau)$ is intuitionistic fuzzy $e$-closed if and only if its complement $A^{c}$ is intuitionistic fuzzy e-open.

Lemma 2.17 ([3]). In any intuitionistic fuzzy topological space X, 
(a) any union of intuitionistic fuzzy e-open sets is an intuitionistic fuzzy e-open set;

(b) any intersection of intuitionistic fuzzy e-closed sets is an intuitionistic fuzzy e-closed set.

Definition $2.18([3])$. Let $(X, \tau)$ be an intuitionistic fuzzy topological space and $A$ be any intuitionistic fuzzy set of $X$. Then the intuitionistic fuzzy e interior of A (in short eint(A)) and intuitionistic fuzzy e closure of $A$ (in short ecl $(A)$ ) are defined as

$$
\begin{aligned}
\operatorname{eint}(A) & =\cup\{G \mid G \text { is an intuitionistic fuzzy e open set in } X \text { and } G \subseteq A\}, \\
\operatorname{ecl}(A) & =\cap\{K \mid K \text { is an intuitionistic fuzzy e closed set inX and } A \subseteq K\} .
\end{aligned}
$$

Remark 2.19 ([3]). If $A$ be an intuitionistic fuzzy set in $X$, then $A \subseteq \operatorname{ecl}(A) \subseteq \operatorname{cl}(A)$.

Proposition 2.20 ([3]). Let $(X, \tau)$ be any intuitionistic fuzzy topological space and $\mathrm{A}$ and $\mathrm{B}$ be any two intuitionistic fuzzy sets in $(X, \tau)$. Then

(a) $\operatorname{ecl}(\tilde{0})=\tilde{0}$ and $\operatorname{ecl}(\tilde{1})=\tilde{1}$;

(b) $A \subseteq \operatorname{ecl}(A) \subseteq \operatorname{cl}(A)$;

(c) $\operatorname{int}(A) \subseteq \operatorname{eint}(A) \subseteq A$;

(d) $A$ is intuitionistic fuzzy e closed if and only if $A=\operatorname{ecl}(A)$;

(e) $A$ is intuitionistic fuzzy e open if and only if $A=\operatorname{eint}(A)$;

(f) $\mathrm{A} \subseteq \mathrm{B} \Rightarrow \operatorname{ecl}(A) \subseteq \operatorname{ecl}(\mathrm{B})$;

(g) $A \subseteq B \Rightarrow \operatorname{eint}(A) \subseteq \operatorname{eint}(B)$.

Definition 2.21 ([35]). An intuitionistic fuzzy set $A$ in an intuitionistic fuzzy topological space $(X, \tau)$ is called

(a) intuitionistic fuzzy generalized closed if $\mathrm{cl}(\mathrm{A}) \subseteq \mathrm{O}$, whenever $\mathrm{A} \subseteq \mathrm{O}$ and $\mathrm{O}$ is an intuitionistic fuzzy open;

(b) intuitionistic fuzzy generalized open if $O \subseteq \operatorname{int}(A)$, whenever $O \subseteq A$ and $O$ is an intuitionistic fuzzy closed.

Remark 2.22 ([30]). Every intuitionistic fuzzy closed (resp. intuitionistic fuzzy open) set is intuitionistic fuzzy g-closed (resp. intuitionistic fuzzy g-open) but the converses may not be true.

\section{Intuitionistic fuzzy generalized e-closed sets}

In this section, we introduce the concept of intuitionistic fuzzy ge-closed set and study some of their properties in intuitionistic fuzzy topological spaces.

Definition 3.1. An intuitionistic fuzzy set $A$ in an intuitionistic fuzzy topological space $(X, \tau)$ is called intuitionistic fuzzy generalized e-closed (written as intuitionistic fuzzy ge-closed) if ecl $(A) \subseteq 0$, whenever $\mathrm{A} \subseteq \mathrm{O}$ and $\mathrm{O}$ is an intuitionistic fuzzy open.

Lemma 3.2. For any two intuitionistic fuzzy sets $A$ and B of $X$,

$$
\rceil\left(A_{q} B\right) \Leftrightarrow A \subset B^{c} .
$$

Remark 3.3. Every intuitionistic fuzzy g-closed set is intuitionistic fuzzy ge-closed but the converse may not be true.

Example 3.4. Let $X=\{a, b\}$ and intuitionistic fuzzy sets $U$ and $A$ on $X$ are defined as follows

$$
\mathrm{U}=\{<\mathrm{a}, 0.2,0.8\rangle,<\mathrm{b}, 0.4,0.6\rangle\}, \quad \mathrm{A}=\{<\mathrm{a}, 0.1,0.8>,<\mathrm{b}, 0.3,0.7\rangle\} .
$$

Let $\tau=\{\tilde{0}, \mathrm{U}, \tilde{1}\}$ be an intuitionistic fuzzy topology on $X$. Then the intuitionistic fuzzy set $A$ is intuitionistic fuzzy ge-closed but not intuitionistic fuzzy $\mathrm{g}$-closed. 
Remark 3.5. Every intuitionistic fuzzy e-closed set is intuitionistic fuzzy ge-closed but the converse may not be true.

Example 3.6. Let $X=\{a, b\}$ and intuitionistic fuzzy sets $U$ and $A$ on $X$ are defined as follows

$$
\mathrm{U}=\{<\mathrm{a}, 0.6,0.4>,<\mathrm{b}, 0.8,0.2>\}, \quad \mathrm{A}=\{<\mathrm{a}, 0.8,0.2>,<\mathrm{b}, 0.9,0.1>\} .
$$

Let $\tau=\{\tilde{0}, \mathrm{U}, \tilde{1}\}$ be an intuitionistic fuzzy topology on $\mathrm{X}$. Then the intuitionistic fuzzy set $\mathrm{A}$ is intuitionistic fuzzy ge-closed but not intuitionistic fuzzy e-closed.

Theorem 3.7. Let $(X, \tau)$ be an intuitionistic fuzzy topological space and $A$ is an intuitionistic fuzzy set of $X$. Then $A$ is intuitionistic fuzzy ge-closed if and only if $\left.\rceil\left(A_{q} F\right) \Rightarrow\right\rceil\left(e c l(A)_{q} F\right)$ for every intuitionistic fuzzy closed set $F$ of $\mathrm{X}$.

Proof.

Necessity: Let $F$ be an intuitionistic fuzzy closed subset of $X$ and $7\left(A_{q} F\right)$. Then by Lemma 3.2, $A \subseteq F^{c}$ and $F^{c}$ intuitionistic fuzzy open in $X$, Therefore ecl $(A) \subseteq F^{c}$ because $A$ is intuitionistic fuzzy ge-closed. Hence by Lemma 3.2, $7\left(\mathrm{ecl}(A)_{\mathrm{q}} \mathrm{F}\right)$.

Sufficiency: Let $O$ be an intuitionistic fuzzy open set of $X$ such that $A \subseteq O$. Then by Lemma 3.2, \rceil$\left(A_{q}\left(O^{c}\right)\right)$, and $O^{c}$ is intuitionistic fuzzy closed in $X$. Hence by hypothesis $\rceil\left(\operatorname{ecl}(A)_{q}\left(O^{c}\right)\right)$. Therefore by Lemma 3.2, $\operatorname{ecl}(A) \subseteq O$. Hence $A$ is intuitionistic fuzzy ge-closed in $X$.

Theorem 3.8. Let $A$ be an intuitionistic fuzzy ge-closed set in an intuitionistic fuzzy topological space $(X, \tau)$ and $c(\alpha, \beta)$ be an Intuitionstic fuzzy point of $X$ such that $c(\alpha, \beta)_{q} \operatorname{ecl}(A)$, then $\operatorname{ecl}(c(\alpha, \beta))_{q} A$.

Proof. If $\rceil \operatorname{ecl}(\operatorname{c}(\alpha, \beta))_{q} A$, then by Lemma 3.2, $A \subseteq(\operatorname{ecl}(\operatorname{c}(\alpha, \beta)))^{c}$ and so ecl $(A) \subseteq(\operatorname{ecl}(\operatorname{cc}(\alpha, \beta)))^{c} \subseteq$ $(c(\alpha, \beta))^{c}$, because $A$ is intuitionistic fuzzy ge-closed set in $X$. Hence by Lemma 3.2, $7\left(c(\alpha, \beta)_{q}(\operatorname{ecl}(A))\right)$, a contradiction.

Remark 3.9. The intersection of two intuitionistic fuzzy ge-closed sets in an intuitionistic fuzzy topological space $(X, \tau)$ may not be intuitionistic fuzzy ge-closed.

Example 3.10. Let $X=\{a, b\}$ and intuitionistic fuzzy sets $U, A$ and $B$ on $X$ are defined as follows:

$\mathrm{U}=\{\langle\mathrm{a}, 0.7,0.2\rangle,\langle\mathrm{b}, 0.6,0.4\rangle\}, \mathrm{A}=\{\langle\mathrm{a}, 0.6,0.4\rangle,\langle\mathrm{b}, 0.7,0.2\rangle\}, \mathrm{B}=\{\langle\mathrm{a}, 0.8,0.2\rangle,<\mathrm{b}, 0.5,0.5\rangle\}$.

Let $\tau=\{\tilde{0}, \mathrm{U}, \tilde{1}\}$ be an intuitionistic fuzzy topology on $\mathrm{X}$. Then $\mathrm{A}$ and $\mathrm{B}$ are the intuitionistic fuzzy ge-closed in $(X, \tau)$ but $A \cap B$ is not intuitionistic fuzzy ge-closed.

Theorem 3.11. Let $A$ be an intuitionistic fuzzy ge-closed sets in an intuitionistic fuzzy topological space $(X, \tau)$ and $\mathrm{A} \subseteq \mathrm{B} \subseteq \mathrm{ecl}(\mathrm{B})$. Then $\mathrm{B}$ is an intuitionistic fuzzy ge-closed in $\mathrm{X}$.

Proof. Let $\mathrm{O}$ be intuitionistic fuzzy open set such that $\mathrm{B} \subseteq \mathrm{O}$. Then $\mathrm{A} \subseteq \mathrm{O}$ and Since $\mathrm{A}$ is intuitionistic fuzzy ge-closed, $\operatorname{ecl}(A) \subseteq \mathrm{O}$. Now $\mathrm{B} \subseteq \operatorname{ecl}(A) \Rightarrow \operatorname{ecl}(B) \subseteq \operatorname{ecl}(A) \subseteq \mathrm{O}$. Consequently $\mathrm{B}$ is intuitionistic fuzzy ge-closed.

Definition 3.12. Let $(X, \tau)$ be an intuitionistic fuzzy topological space and $Y$ be a crisp subset of $X$. Then the induced intuitionistic fuzzy topology (relative intuitionistic fuzzy topology) $\tau_{Y}$ for $Y$ is defined by $\tau_{Y}=\{Y \cap O: O \in \tau\}$. The pair $\left(Y, \tau_{Y}\right)$ is called a subspace of $(X, \tau)$.

Lemma 3.13. Let $\left(\mathrm{Y}, \tau_{\mathrm{Y}}\right)$ be a subspace of an intuitionistic fuzzy topological space $(\mathrm{X}, \tau)$ and $\mathrm{A}$ be an intuitionistic fuzzy set of $\mathrm{Y}$. Then

(a) $A$ is $\tau_{Y}$-intuitionistic fuzzy closed if and only if there exists an intuitionistic fuzzy open set $\mathrm{F}$ in $\mathrm{X}$ such that $A=F \cap Y$; 
(b) $\operatorname{cl}_{Y}(A)=Y \cap \operatorname{cl}(A)$;

(c) $\operatorname{ecl}_{Y}(A)=\mathrm{Y} \cap \operatorname{ecl}(A)$, if $Y$ is intuitionistic fuzzy regular open.

Proof. Obvious.

Theorem 3.14. Let $\left(Y, \tau_{Y}\right)$ be a intuitionistic fuzzy regular open subspace of an intuitionistic fuzzy topological space $(X, \tau)$ and $\mathrm{A}$ be an intuitionistic fuzzy set in $\mathrm{Y}$. If $\mathrm{A}$ is intuitionistic fuzzy ge-closed in $\mathrm{X}$, then $\mathrm{A}$ is intuitionistic fuzzy ge-closed in $\mathrm{Y}$.

Proof. Let $A \subseteq O_{Y}$, where $O_{Y}$ is an intuitionistic fuzzy open in $Y$. Then by Lemma 3.2 there exists an intuitionistic fuzzy open set $O$ in $X$ such that $O_{Y}=O \cap Y$. Therefore $A \subseteq O$ and since $A$ is intuitionistic fuzzy ge-closed in $X, \operatorname{ecl}(A) \subseteq \mathrm{O}$. It follows that $\operatorname{ecl}_{Y}(A)=\operatorname{ecl}(A) \cap Y \subset \mathrm{O} \cap \mathrm{Y}=\mathrm{O}_{Y}, \mathrm{Y}$ is intuitionistic fuzzy regular open. Hence $A$ is intuitionistic fuzzy ge-closed in $Y$.

Theorem 3.15. Let $(X, \tau)$ be an intuitionistic fuzzy topological space and $\mathfrak{R}$ be the family of all intuitionistic fuzzy $e$-closed sets of $X$. Then $\tau=\mathfrak{R}$ if and only if every intuitionistic fuzzy subset of $X$ is intuitionistic fuzzy ge-closed.

Proof.

Necessity: Suppose that $\tau=\Re$ and such that $A \subseteq \mathrm{O} \in \tau$ then $\operatorname{ecl}(A) \subseteq \operatorname{ecl}(O)=O$ and $A$ is intuitionistic fuzzy ge-closed.

Sufficiency: Suppose that every intuitionistic fuzzy set of $X$ is intuitionistic fuzzy ge-closed. Let $O \in \tau$ then since $\mathrm{O} \subseteq \mathrm{O}$ and $\mathrm{O}$ is intuitionistic fuzzy ge-closed, where $\operatorname{ecl}(\mathrm{O}) \subseteq \mathrm{O}$ and $\mathrm{O} \in \mathfrak{R}$. Thus $\tau \subseteq \mathfrak{R}$. If $T \in \mathfrak{R}$, then $T^{c} \in \tau \subseteq \mathfrak{R}$, and hence $T \in \tau$ consequently $\mathfrak{R} \subseteq \tau$ and $\tau=\mathfrak{R}$.

Definition 3.16. An intuitionistic fuzzy set $A$ in an intuitionistic fuzzy topological space $(X, \tau)$ is called intuitionistic fuzzy ge-open if and only if $A^{c}$ is intuitionistic fuzzy ge-closed.

Remark 3.17. Every intuitionistic fuzzy open set is intuitionistic fuzzy ge-open. But the converse may not be true. For the intuitionistic fuzzy set $C=<X,(0.6,0.6),(0.3,0.4)>$ in the intuitionistic fuzzy topological space $(X, \tau)$ of Example 3.6 is intuitionistic fuzzy ge-open but not intuitionistic fuzzy open.

Theorem 3.18. An intuitionistic fuzzy set $\mathrm{A}$ of an intuitionistic fuzzy topological space $(X, \tau)$ is intuitionistic fuzzy ge-open if and only if $\mathrm{F} \subseteq \operatorname{eint}(A)$ whenever $\mathrm{F}$ is intuitionistic fuzzy closed and $\mathrm{F} \subseteq \mathrm{A}$.

Proof. Suppose $A$ is intuitionistic fuzzy ge-open in $X$ and $\operatorname{eint}(A) \subseteq B \subseteq A$. Then $A^{c}$ is intuitionistic fuzzy ge-closed and $A^{c} \subseteq B^{c} \subseteq \operatorname{ecl}\left(A^{c}\right)$. Therefore by Theorem 3.7, $\mathrm{B}^{c}$ is intuitionistic fuzzy ge-closed in $X$, hence $B$ is intuitionistic fuzzy ge-open in $X$.

Remark 3.19. The union of two intuitionistic fuzzy ge-open sets in an intuitionistic fuzzy topological space $(X, \tau)$ may not be intuitionistic fuzzy ge-open.

Example 3.20. Let $X=\{a, b\}$ and intuitionistic fuzzy sets $U, C$ and $D$ on $X$ are defined as follows $\mathrm{U}=\{\langle\mathrm{a}, 0.7,0.2\rangle,\langle\mathrm{b}, 0.6,0.4\rangle\}, \mathrm{C}=\{\langle\mathrm{a}, 0.4,0.6\rangle,<\mathrm{b}, 0.2,0.7\rangle\}, \mathrm{D}=\{\langle\mathrm{a}, 0.2,0.8\rangle,\langle\mathrm{b}, 0.5,0.5\rangle\}$.

Let $\tau=\{\tilde{0}, \mathrm{U}, \tilde{1}\}$ be an intuitionistic fuzzy topology on $X$. Then $\mathrm{C}$ and $\mathrm{D}$ are the intuitionistic fuzzy ge-open in $(X, \tau)$ but $A \cup B$ is not intuitionistic fuzzy ge-open.

Theorem 3.21. Let $A$ be an intuitionistic fuzzy ge-open subsets of an intuitionistic fuzzy topological space $(X, \tau)$ and $\operatorname{eint}(A) \subseteq B \subseteq A$. Then $B$ is an intuitionistic fuzzy ge-open in $\mathrm{X}$.

Proof. Since $A^{c} \subseteq B^{c} \subseteq \operatorname{ecl}\left(A^{c}\right)$ and $A^{c}$ is intuitionistic fuzzy ge-closed, it follows that $B^{c}$ is intuitionistic fuzzy ge-closed by Theorem 3.11, thus $B$ is intuitionistic fuzzy ge-open.

Theorem 3.22. If $A$ is an intuitionistic fuzzy e-open and intuitionistic fuzzy ge-closed in an intuitionistic fuzzy topological space $(X, \tau)$, then $A$ is an intuitionistic fuzzy e-closed in $(X, \tau)$.

Proof. Since $A$ is an intuitionistic fuzzy e-open and intuitionistic fuzzy gege-closed in $(X, \tau), \operatorname{ecl}(A) \subseteq A$, but $A \subseteq \operatorname{ecl}(A)$. This implies $\operatorname{ecl}(A)=A$ and hence $A$ is an intuitionistic fuzzy e-closed in $(X, \tau)$. 


\section{Intuitionistic fuzzy GEO-connectedness and intuitionistic fuzzy GEO-compactness}

Definition 4.1. An intuitionistic fuzzy topological space $(X, \tau)$ is said to be intuitionistic fuzzy GEOconnected if the only intuitionistic fuzzy sets which are both intuitionistic fuzzy ge-open and intuitionistic fuzzy ge-closed are $\tilde{0}$ and $\tilde{1}$.

Theorem 4.2. Every intuitionistic fuzzy GEO-connected space is intuitionistic fuzzy $\mathrm{C}_{5}$-connected.

Proof. Let $(X, \tau)$ be an intuitionistic fuzzy GEO-connected space and suppose that $(X, \tau)$ sace is not intuitionistic fuzzy $C_{5}$-connected. Then there exists a proper intuitionistic fuzzy set $A=\{A \neq \tilde{0}, A \neq \tilde{1}\}$ such that $A$ is both intuitionistic fuzzy e-open and intuitionistic fuzzy e-closed. Since every intuitionistic fuzzy e-open (resp. intuitionistic fuzzy e-closed) set is intuitionistic fuzzy ge-open (resp. intuitionistic fuzzy ge-closed), $X$ is not intuitionistic fuzzy GEO-connected, a contradiction.

Remark 4.3. The converse of the Theorem 4.2 may be false.

Example 4.4. Let $X=\{a, b\}$ and intuitionistic fuzzy sets $U$ are defined as follows:

$$
\mathrm{U}=\{<\mathrm{a}, 0.5,0.5>,<\mathrm{b}, 0.7,0.3>\} .
$$

Let $\tau=\{\tilde{0}, \mathrm{U}, \tilde{1}\}$ be an intuitionistic fuzzy topology on $X$. Then $(X, \tau)$ intuitionistic fuzzy $C_{5}$-connected but not intuitionistic fuzzy GEO-connected.

Theorem 4.5. An intuitionistic fuzzy topological space $(X, \tau)$ is intuitionistic fuzzy GEO-connected if and only if there exists no non zero intuitionistic fuzzy ge-open sets $\mathrm{A}$ and $\mathrm{B}$ in $\mathrm{X}$ such that $\mathrm{A}=\mathrm{B}^{\mathrm{c}}$.

Proof.

Necessity: Suppose that $A$ and $B$ are intuitionistic fuzzy ge-open sets in $X$ such that $A \neq \tilde{0} \neq B$ and $A=B^{c}$. Since $A=B^{c}, B$ is an intuitionistic fuzzy ge-closed set and $A \neq \tilde{0} \Rightarrow B \neq \tilde{1}$. But this is a contradiction to the fact that $X$ is intuitionistic fuzzy GEO-connected.

Sufficiency: Let $A$ be both an intuitionistic fuzzy ge-open set and intuitionistic fuzzy ge-closed set such that $\tilde{0} \neq A \neq \tilde{1}$. Now take $B=A^{c}$. In this case $B$ is an intuitionistic fuzzy ge-open set and $A \neq \tilde{1} \Rightarrow B=$ $A^{c} \neq \tilde{0}$, which is a contradiction.

Definition 4.6. Let $(X, \tau)$ be an intuitionistic fuzzy topological space and $A$ be an intuitionistic set in $X$. Then the ge-interior and ge-closure of $A$ are defined as follows:

$$
\begin{aligned}
\operatorname{geint}(A) & =\cup\{G \mid G \text { is an intuitionistic fuzzy ge-open set in } X \text { and } G \subseteq A\}, \\
\operatorname{gecl}(A) & =\cap\{K \mid K \text { is an intuitionistic fuzzy ge-closed set in } X \text { and } A \subseteq K\} .
\end{aligned}
$$

Remark 4.7. It is clear that, $A \subseteq \operatorname{gecl}(A) \subseteq \operatorname{cl}(A)$ for any intuitionistic fuzzy set $A$ of $X$.

Theorem 4.8. An intuitionistic fuzzy topological space $(X, \tau)$ is intuitionistic fuzzy GEO-connected if and only if there exists no non zero intuitionistic fuzzy ge-open sets $\mathrm{A}$ and $\mathrm{B}$ in $\mathrm{X}$ such that $\mathrm{B}=\mathrm{A}^{\mathrm{c}}, \mathrm{B}=(\operatorname{gecl}(\mathrm{A}))^{\mathrm{c}}$, $\mathrm{A}=(\operatorname{gecl}(\mathrm{B}))^{\mathrm{c}}$.

Proof.

Necessity: Assume that there exists intuitionistic fuzzy sets $A$ and $B$ such that $A \neq 0 \neq B$ and $B=A^{c}$, $B=(\operatorname{gecl}(A))^{c}, A=(\operatorname{gecl}(B))^{c}$. Since $(\operatorname{gecl}(A))^{c}$, and $(\operatorname{gecl}(B))^{c}$ are intuitionistic fuzzy ge-open sets in $X$, $A$ and $B$ are intuitionistic fuzzy ge-open sets in $X$, which is contradiction.

Sufficiency: Let $A$ be both an intuitionistic fuzzy ge-open set and intuitionistic fuzzy ge-closed set such that $\tilde{0} \neq A \neq \tilde{1}$. Now take $B=A^{c}$, we obtain a contradiction. 
Definition 4.9. An intuitionistic fuzzy topological space $(X, \tau)$ is said to be intuitionistic fuzzy $e T_{1 / 2}$ if every intuitionistic fuzzy ge-closed set in $X$ is intuitionistic fuzzy e-closed in $X$.

Theorem 4.10. Let $(\mathrm{X}, \tau)$ be an intuitionistic fuzzy $\mathrm{e}_{1 / 2}$ space, then the following conditions are equivalent:

(a) $X$ is intuitionistic fuzzy GEO-connected;

(b) $\mathrm{X}$ is intuitionistic fuzzy $\mathrm{C}_{5}$-connected.

Proof.

(a) $\Rightarrow$ (b) Follows from Theorem 4.2.

(b) $\Rightarrow$ (a) Let $X$ is intuitionistic fuzzy $e T_{1 / 2}$ and intuitionistic fuzzy $C_{5}$-connected. If possible, let $X$ is not intuitionistic fuzzy GEO-connected, then there exists a proper intuitionistic fuzzy set $A$ such that $A$ is both intuitionistic fuzzy ge-open set and intuitionistic fuzzy ge-closed. Since $X$ is intuitionistic fuzzy $e T_{1 / 2}, A$ is intuitionistic fuzzy e-open and intuitionistic fuzzy e-closed, which implies that $X$ is not intuitionistic fuzzy $\mathrm{C}_{5}$-connected, a contradiction.

Definition 4.11. An intuitionistic fuzzy ge-open set $A$ is called intuitionistic fuzzy regular ge-open if $A=\operatorname{geint}(\operatorname{gecl}(A))$. The complement of an intuitionistic fuzzy regular ge-open set is called intuitionistic fuzzy regular ge-closed.

Definition 4.12. An intuitionistic fuzzy topological space $(X, \tau)$ is called intuitionistic fuzzy GEO-super connected if there is no proper intuitionistic fuzzy regular ge-open in $X$.

Theorem 4.13. Let $(X, \tau)$ be an intuitionistic fuzzy topological space, then the following conditions are equivalent.

(a) $X$ is intuitionistic fuzzy GEO-super connected.

(b) For every non zero intuitionistic fuzzy ge-open set $A$, $\operatorname{gecl}(A)=\tilde{1}$.

(c) For every intuitionistic fuzzy ge-closed set $A$ with $A \neq \tilde{1}$, geint $(A)=\tilde{0}$.

(d) There exists no intuitionistic fuzzy ge-open set $\mathrm{A}$ and $\mathrm{B}$ in $\mathrm{X}$ such that $\mathrm{A} \neq 0$ $\neq \mathrm{B}, \mathrm{A} \subseteq \mathrm{B}^{\mathrm{c}}$.

(e) There exists no intuitionistic fuzzy ge-open set $\mathrm{A}$ and $\mathrm{B}$ in $\mathrm{X}$ such that $\mathrm{A} \neq \tilde{0} \neq \mathrm{B}, \mathrm{B}=(\operatorname{gecl}(\mathrm{A}))^{\mathrm{c}}$, $A=(\operatorname{gecl}(B))^{c}$.

(f) There exists no intuitionistic fuzzy ge-closed set $\mathrm{A}$ and $\mathrm{B}$ in $\mathrm{X}$ such that $\mathrm{A} \neq \tilde{1} \neq \mathrm{B}, \mathrm{B}=(\operatorname{geint}(\mathrm{A}))^{\mathrm{c}}$, $A=(\operatorname{geint}(B))^{c}$.

Proof.

$(a) \Rightarrow(b)$ Assume that there exists an intuitionistic fuzzy ge-open $A \neq \tilde{0}$ such that $\operatorname{gecl}(A) \neq \tilde{1}$. Now we take $B=\operatorname{geint}(\operatorname{gecl}(A))^{c}$. Then $B$ is proper intuitionistic fuzzy regular ge-open in $X$, and this is a contradiction with the intuitionistic fuzzy GEO-super connectedness of $X$.

(b) $\Rightarrow$ (c) Let $A \neq \tilde{1}$ be an intuitionistic fuzzy ge-closed set in $X$. If we take $B \subseteq A^{c}$, then $B$ is an intuitionistic fuzzy ge-open set in $X$, and $B \neq \tilde{0}$. Hence $\operatorname{gecl}(B)=\tilde{1} \Rightarrow(\operatorname{gecl}(B))^{c}=\tilde{0} \Rightarrow \operatorname{geint}(B)^{c}=\tilde{0} \Rightarrow$ $\operatorname{geint}(A)=\tilde{0}$ follows.

(c) $\Rightarrow(d)$ Let $A$ and $B$ be any two intuitionistic fuzzy ge-open sets in $X$ such that $A \neq \tilde{0} \neq B$ and $A \subseteq B^{c}$. Since $B^{c}$ is an intuitionistic fuzzy ge-closed set in $X$ and $B \neq \tilde{0} \Rightarrow B^{c} \neq \tilde{1}$, we obtain geint $(B)^{c}=\tilde{0}$. But, from $A \subseteq B^{c}$, we see that $\tilde{0} \neq A=\operatorname{geint}(A) \subseteq \operatorname{geint}(B)^{c}=\tilde{0}$, which is a contradiction.

$(d) \Rightarrow(a)$ Let $\tilde{0} \neq A \neq \tilde{1}$, any intuitionistic fuzzy regular ge-open set in $X$. If we take $B=(\operatorname{gecl}(A))^{c}$, we get $B \neq \tilde{0}$ (because, otherwise we have $B=\tilde{0} \Rightarrow(\operatorname{gecl}(A))^{c}=\tilde{0} \Rightarrow \operatorname{gecl}(A)=\tilde{1} \Rightarrow A=B=$ $\operatorname{geint}(\operatorname{gecl}(A))=\operatorname{geint}(\tilde{1})=\tilde{1}$, but the last result contradicts the fact $A \neq \tilde{1})$. We also have $A \subseteq B^{c}$, and this is a contradiction, too.

$(a) \Rightarrow(e)$ Let $A$ and $B$ be any two intuitionistic fuzzy regular ge-open sets in $X$ such that $A \neq \tilde{0} \neq B$ and $B=(\operatorname{gecl}(A))^{c}, A=(\operatorname{gecl}(B))^{c}$. Now we have $\operatorname{geint}(\operatorname{gecl}(A))=\operatorname{geint}\left(B^{c}\right)=(\operatorname{gecl}(B))^{c}=A$ and $A \neq \tilde{0}, A \neq \tilde{1}$ (if not, i.e., if $A=\tilde{1}$, then $\left.\tilde{1}=(\operatorname{gecl}(B))^{c} \Rightarrow \tilde{0}=\operatorname{gecl}(B) \Rightarrow B=\tilde{0}\right)$. But this is a contradiction. 
$(e) \Rightarrow(a)$ Let $A$ and $B$ be any two intuitionistic fuzzy regular ge-open sets in $X$ such that $A=\operatorname{geint}(\operatorname{gecl}(A)), \tilde{0} \neq A \neq \tilde{1}$. Now we take $B=(\operatorname{gecl}(A))^{c}$. In this case we get $B \neq \tilde{0}$ and $B$ is intuitionistic fuzzy regular ge-open sets in $X . B=(\operatorname{gecl}(A))^{c},(\operatorname{gecl}(B))^{c}=\left(\operatorname{gecl}(\operatorname{gecl}(A))^{c}\right)^{c}=(\operatorname{geint}(\operatorname{gecl}(A)))^{\mathfrak{c}}=$ $\operatorname{geint}(\operatorname{gecl}(A))=A$, which is a contradiction.

$(e) \Rightarrow(f)$ Let $A$ and $B$ be any two intuitionistic fuzzy regular ge-closed sets in $X$ such that $A \neq \tilde{1} \neq B$, $\mathrm{B}=(\operatorname{geint}(A))^{\mathrm{c}}, \mathrm{A}=(\operatorname{geint}(\mathrm{B}))^{\mathrm{c}}$. Taking $\mathrm{C}=\mathrm{A}^{\mathrm{c}}$ and $\mathrm{D}=\mathrm{B}^{\mathrm{c}}, \mathrm{C}$ and $\mathrm{D}$ become intuitionistic fuzzy regular ge-open sets in $X$ and $C \neq \tilde{0} \neq D,(\operatorname{gecl}(C))^{c}=\left(\operatorname{gecl}\left(A^{c}\right)\right)^{c}=\left(\operatorname{geint}(A)^{c}\right)^{c}=\operatorname{geint}(A)=B^{c}=D$, and similarly $(\operatorname{gecl}(D))^{\mathrm{c}}=\mathrm{C}$. But this is an obvious contradiction.

$(f) \Rightarrow(e)$ One can use a similar technique as in (e) $\Rightarrow(f)$.

Definition 4.14. An intuitionistic fuzzy topological space $(X, \tau)$ is called intuitionistic fuzzy GEO-extremely disconnected, if the ge-closure of every intuitionistic fuzzy ge-open set in $\mathrm{X}$ is intuitionistic fuzzy ge-open.

Theorem 4.15. Let $(X, \tau)$ be an intuitionistic fuzzy topological space, then the following conditions are equivalent.

(a) $X$ is intuitionistic fuzzy GEO-extremely disconnected.

(b) For each intuitionistic fuzzy ge-closed set $A$, geint $(A)$ is intuitionistic fuzzy ge-closed.

(c) For each intuitionistic fuzzy ge-open set $A, \operatorname{gecl}(A)=\left[\operatorname{gecl}(\operatorname{gecl}(A))^{c}\right]^{c}$.

(d) For each pair of intuitionistic fuzzy ge-open set $\mathrm{A}$ and $\mathrm{B}$ with $\operatorname{gecl}(\mathrm{A})=\mathrm{B}^{\mathrm{c}}$, $\operatorname{gecl}(\mathrm{A})=(\operatorname{gecl}(\mathrm{B}))^{\mathrm{c}}$.

Proof.

(a) $\Rightarrow$ (b) Let $A$ be any intuitionistic fuzzy ge-closed set. Then $A^{c}$ is intuitionistic fuzzy ge-open and so by $($ a $) \operatorname{gecl}\left(A^{c}\right)=(\operatorname{geint}(A))^{c}$ is intuitionistic fuzzy ge-open, which implies that geint $(A)$ is intuitionistic fuzzy ge-closed.

(b) $\Rightarrow(\mathrm{c})$ Let $A$ be any intuitionistic fuzzy ge-open set, we have $\operatorname{gecl}(\operatorname{gecl}(A))^{\mathfrak{c}}=\operatorname{gecl}\left(\operatorname{geint}\left(A^{\mathfrak{c}}\right)\right) \Rightarrow$ $\left[\operatorname{gecl}\left(\operatorname{gecl}(A)^{\mathfrak{c}}\right)\right]^{\mathfrak{c}}=\left[\operatorname{gecl}\left(\operatorname{geint}\left(A^{\mathfrak{c}}\right)\right)\right]$, since $A$ is intuitionistic fuzzy ge-open, $A^{\mathfrak{c}}$ is intuitionistic fuzzy geclosed and so by (b), geint $\left(A^{c}\right)$ is intuitionistic fuzzy ge-closed, that is $\operatorname{gecl}\left(\operatorname{geint}\left(A^{\mathfrak{c}}\right)\right)=\operatorname{geint}\left(A^{\mathfrak{c}}\right)$, thus we have $\left[\operatorname{gecl}\left(\operatorname{geint}\left(A^{\mathfrak{c}}\right)\right)\right]^{\mathfrak{c}}=\left[\operatorname{geint}\left(A^{\mathfrak{c}}\right)\right]^{\mathfrak{c}}=\operatorname{gecl}(A)$.

(c) $\Rightarrow$ (d) Let $A$ and $B$ be any two intuitionistic fuzzy ge-open sets in $X$ such that $\operatorname{gecl}(A)=B^{c}$. Then by $(\mathrm{c})$, we have $\operatorname{gecl}(A)=\left[\operatorname{gecl}\left(\operatorname{gecl}\left(A^{\mathrm{c}}\right)\right)\right]^{\mathrm{c}} \Rightarrow\left[\operatorname{gecl}\left(B^{\mathrm{c}}\right)^{\mathrm{c}}\right]^{\mathrm{c}}=[\operatorname{gecl}(B)]^{\mathrm{c}}$.

$(d) \Rightarrow(a)$ Let $A$ be any intuitionistic fuzzy ge-open set. Put $B=(\operatorname{gecl}(A))^{c}$. Then $(\operatorname{gecl}(A))^{c}=B^{c}$, so by (d) $\operatorname{gecl}(A)=[\operatorname{gecl}(B)]^{c}$, since $\operatorname{gecl}(B)$ is intuitionistic fuzzy ge-closed it follows that $\operatorname{gecl}(A)=[\operatorname{gecl}(B)]^{c}$ is intuitionistic fuzzy ge-open.

Definition 4.16. A Collection $\left\{A_{i}: i \in \Lambda\right\}$ of intuitionistic fuzzy ge-open sets in an intuitionistic fuzzy topological space $(X, \tau)$ is called an intuitionistic fuzzy ge-open cover of an intuitionistic fuzzy set $B$ of $X$ if $B \subseteq \cup\left\{A_{i}: i \in \Lambda\right\}$.

Definition 4.17. An intuitionistic fuzzy topological space $(X, \tau)$ is called intuitionistic fuzzy GEO-compact if every intuitionistic fuzzy ge-open cover of $X$ has a finite subcover.

Definition 4.18. An intuitionistic fuzzy set $B$ of an intuitionistic fuzzy topological space $(X, \tau)$ is called intuitionistic fuzzy GEO-compact relative to $X$, if for every collection $\left\{A_{i}: i \in \Lambda\right\}$ of intuitionistic fuzzy ge-open subsets of $X$ such that $B \subseteq \cup\left\{A_{i}: i \in \Lambda\right\}$, there exists a finite subset $\Lambda_{0}$ of $\Lambda$ such that $B \subseteq \cup\left\{A_{i}\right.$ : $i \in \Lambda$ \}.

Definition 4.19. A crisp subset $B$ of an intuitionistic fuzzy topological space $(X, \tau)$ is called intuitionistic fuzzy GEO-compact if B is intuitionistic fuzzy GEO-compact as an intuitionistic fuzzy subsets of $X$.

Theorem 4.20. An intuitionistic fuzzy ge-closed crisp subset of an intuitionistic fuzzy GEO-compact space $(X, \tau)$ is an intuitionistic fuzzy GEO-compact relative to X. 
Proof. Let $A$ be an intuitionistic fuzzy ge-closed crisp set of an intuitionistic fuzzy GEO-compact space $(X, \tau)$. Then $A^{c}$ is intuitionistic fuzzy ge-open in $X$. Let $M$ be a cover of $A$ by intuitionistic fuzzy geopen sets in $X$. Then $\left\{M, A^{c}\right\}$ is an intuitionistic fuzzy ge-open cover of $X$. Since $X$ is intuitionistic fuzzy GEO-compact, it has a finite subcover. If this subcover contain $A^{c}$, we discard it. Otherwise leave the subcover as it is, thus we have obtained a finite intuitionistic fuzzy ge-open subcover of $A$. Therefore $A$ is intuitionistic fuzzy GEO-compact relative to $X$.

Theorem 4.21. Let $(X, \tau)$ be an intuitionistic fuzzy GEO-compact space and $\mathrm{Y}$ is an intuitionistic fuzzy ge-closed crisp subset of $\mathrm{X}$, then $\left(\mathrm{Y}, \tau_{\mathrm{Y}}\right)$ is intuitionistic fuzzy GEO-compact.

Proof. Let $\Upsilon$ be a $\tau_{Y}$-intuitionistic fuzzy e-open covering of $Y$ and let $\{G=V \in G E O(X): V \cap Y \in \Upsilon\}$, then $\mathrm{Y} \subseteq \cup \mathrm{G}$. Since $\mathrm{Y}$ is intuitionistic fuzzy ge-closed, ecl $(\mathrm{Y}) \subseteq \cup \mathrm{G}$. Therefore $\mathrm{G} \cup(\operatorname{ecl}(\mathrm{Y}))^{\mathrm{c}}$ is a $\tau$-intuitionistic fuzzy e-open cover of $X$. Since $X$ is intuitionistic fuzzy GEO-compact, $G \cup(\operatorname{ecl}(Y))^{c}$ has a finite subcover $\left\{V_{1}, V_{2}, \ldots, V_{n},(\operatorname{ecl}(Y))^{c}\right\}$. But then, $\left\{V_{1} \cap Y, V_{2} \cap Y, \ldots, V_{n} \cap Y\right\}$ is a finite subcover of $r$.

Theorem 4.22. An intuitionistic fuzzy topological space $(X, \tau)$ is intuitionistic fuzzy GEO-compact if and only if every family of intuitionistic fuzzy ge-closed sets of $\mathrm{X}$ which has the finite intersection property has a non empty intersection.

Proof.

Necessity: Let $A=\left\{A_{i}: i \in \Lambda\right\}$ be any family of intuitionistic fuzzy ge-closed subset of $X$ with finite intersection property. Then the collection $B=\left\{A_{i}^{c}: i \in \Lambda\right\}$ is intuitionistic fuzzy ge-open cover of $X$. We assert that no finite subfamily of $B$ covers $X$. Let $G=\left\{A_{k}^{c}: k=1,2,3, \ldots, n\right\}$ be any non empty finitely subfamily of $H$. Then $\left\{\cup\left\{A_{k}: k=1,2,3, \ldots, n\right\}\right\}^{c}=\cap\left\{A_{k}: k=1,2,3, \ldots, n\right\} \neq \tilde{0}$. Since by the hypothesis $A$ has the finite intersection property. This implies that $\left\{\cup\left\{A_{k}: k=1,2,3, \ldots, n\right\}\right\}^{c} \neq \tilde{1}$. Since $X$ is intuitionistic fuzzy GEO-compact, $G$ does cover $X$. Hence $\left\{\cup\left\{A_{k}: k=1,2,3, \ldots, n\right\}\right\}^{c} \neq \tilde{1}$. Consequently we obtain that $\left\{\cap\left\{A_{k}: k=1,2,3, \ldots, n\right\}\right\}^{c} \neq \tilde{1}$. Thus $\left\{\cap\left\{A_{k}: k=1,2,3, \ldots, n\right\}\right\}^{c} \neq \tilde{0}$.

Sufficiency: Suppose that every family of intuitionistic fuzzy ge-closed subset of $X$ with finite intersection property has non empty intersection. Let $\left\{A_{k}: k \in \Lambda\right\}$ be intuitionistic fuzzy GEO-cover of X. Thus $\left\{A_{k}: k \in \Lambda\right\}^{c}$ is a family of ge-closed sets in $X$, whose intersection is empty. Therefore, by assumption $\left\{A_{k}: k \in \Lambda\right\}^{c}$ does not possess the finite intersection property and so, there is a finite subfamily say $\left\{A_{k}^{c}: k=1,2,3, \ldots, n\right\}$ of $\left\{A_{k}: k \in \Lambda\right\}^{c}$ with empty intersection. This implies $\left\{A_{k}: k=1,2,3, \ldots, n\right\}=\tilde{1}$. It follows that $X$ is intuitionistic fuzzy GEO-compact.

\section{Intuitionistic fuzzy generalized e-continuity}

The purpose of the present section is to extend and study of ge-continuous mappings in intuitionistic fuzzy topological spaces.

Definition 5.1. A mapping $f:(X, \tau) \rightarrow(Y, \sigma)$ is said to be intuitionistic fuzzy ge-continuous if the inverse image of every intuitionistic fuzzy closed set of $Y$ is intuitionistic fuzzy ge-closed in $X$.

Remark 5.2. Every intuitionistic fuzzy continuous mapping is intuitionistic fuzzy g-continuous, but the converse may not be true.

Remark 5.3. Every intuitionistic fuzzy continuous mapping is intuitionistic fuzzy ge-continuous, but the converse may not be true.

Example 5.4. Let $X=\{a, b\}, Y=\{p, q\}$ and intuitionistic fuzzy sets $U$ and $V$ are defined as follows

$$
\mathrm{U}=\{<\mathrm{a}, 0.5,0.4>,<\mathrm{b}, 0.6,0.4>\}, \quad \mathrm{V}=\{<\mathrm{p}, 0.3,0.6>,<\mathrm{q}, 0.4,0.6>\} .
$$

Let $\tau=\{\tilde{0}, \mathrm{U}, \tilde{1}\}$ and $\sigma=\{\tilde{0}, \mathrm{~V}, \tilde{1}\}$ be intuitionistic fuzzy topologies on $\mathrm{X}$ and $\mathrm{Y}$, respectively. Then the mapping $f:(X, \tau) \rightarrow(Y, \sigma)$ defined by $f(a)=p$ and $f(b)=q$ is intuitionistic fuzzy ge-continuous but not intuitionistic fuzzy continuous. 
Theorem 5.5. A mapping $\mathrm{f}:(\mathrm{X}, \tau) \rightarrow(\mathrm{Y}, \sigma)$ is intuitionistic fuzzy ge-continuous if and only if the inverse image of every intuitionistic fuzzy open set of $\mathrm{Y}$ is intuitionistic fuzzy ge-open in $\mathrm{X}$.

Proof. It is obvious because $f^{-1}\left(U^{c}\right)=\left(f^{-1}(U)\right)^{c}$ for every intuitionistic fuzzy set $U$ of $Y$.

Theorem 5.6. If $\mathrm{f}:(\mathrm{X}, \tau) \rightarrow(\mathrm{Y}, \sigma)$ is intuitionistic fuzzy ge-continuous then for each intuitionistic fuzzy point $c(\alpha, \beta)$ of $X$ and each intuitionistic fuzzy open set $V, f(c(\alpha, \beta)) \subseteq V$ there exist a intuitionistic fuzzy ge-open set $\mathrm{U}$ such that $\mathrm{c}(\alpha, \beta) \subseteq \mathrm{U}$ and $\mathrm{f}(\mathrm{U}) \subseteq \mathrm{V}$.

Proof. Let $c(\alpha, \beta)$ be a intuitionistic fuzzy point of $X$ and $V$ be a intuitionistic fuzzy open set such that $c(\alpha, \beta) \subseteq V$, put $U=f^{-1}(V)$ then by hypothesis $U$ is intuitionistic fuzzy ge-open set of $X$ such that $c(\alpha, \beta) \subseteq U$ and $f(U)=f\left(f^{-1}(V)\right) \subseteq V$.

Theorem 5.7. If $f:(X, \tau) \rightarrow(Y, \sigma)$ is intuitionistic fuzzy ge-continuous then for each intuitionistic fuzzy point $c(\alpha, \beta)$ in $X$ and each intuitionistic fuzzy open set $V$ of $Y$ such that $c(\alpha, \beta)_{q} V$, there exist $c(\alpha, \beta)$ in an intuitionistic fuzzy ge-open set $\mathrm{U}$ of $\mathrm{X}$ such that $\mathrm{c}(\alpha, \beta)_{\mathrm{q}} \mathrm{U}$ and $\mathrm{f}(\mathrm{U}) \subseteq \mathrm{V}$.

Proof. Let $c(\alpha, \beta)$ be an intuitionistic fuzzy point of $X$ and $V$ be an intuitionistic fuzzy open set of $Y$ such that $f(c(\alpha, \beta))_{q} V$. Put $U=f^{-1}(V)$. Then by hypothesis $U$ is an intuitionistic fuzzy ge-open set of $X$ such that $c(\alpha, \beta)_{q} U$ and $f(U)=f\left(f^{-1}(V)\right) \subseteq V$.

Theorem 5.8. If $\mathrm{f}:(X, \tau) \rightarrow(\mathrm{Y}, \sigma)$ is an intuitionistic fuzzy ge-continuous, then $\mathrm{f}(\operatorname{gecl}(A)) \subseteq \operatorname{ecl}(f(A))$ for every intuitionistic fuzzy set $\mathrm{A}$ of $\mathrm{X}$.

Proof. Let $A$ be an intuitionistic fuzzy set of $X$. Then ecl $(f(A))$ is an intuitionistic fuzzy closed set of $Y$. Since $f$ is intuitionistic fuzzy ge-continuous, $f^{-1}(\operatorname{ecl}(f(A)))$ is intuitionistic fuzzy ge-closed in $X$. Clearly $A \subseteq f^{-1}(\operatorname{ecl}(f(A)))$. Therefore $\operatorname{gecl}(A) \subseteq \operatorname{gecl}\left(f^{-1}(\operatorname{ecl}(f(A)))\right)=f^{-1}(\operatorname{ecl}(f(A)))$. Hence $f(\operatorname{gecl}(A)) \subseteq$ $\operatorname{ecl}(f(a))$.

Remark 5.9. The converse of Theorem 5.8 may not be true.

Example 5.10. Let $X=\{a, b, c\}, Y=\{p, q, r\}$ and intuitionistic fuzzy sets $U$ and $V$ are defined as follows

$$
\mathrm{U}=\{\langle\mathrm{a}, 1,0\rangle,\langle\mathrm{b}, 0,1\rangle,\langle\mathrm{c}, 0,1\rangle\}, \quad \mathrm{V}=\{\langle\mathrm{p}, 1,0\rangle,<\mathrm{q}, 0,1\rangle,<\mathrm{r}, 1,0\rangle\}
$$

Let $\tau=\{\tilde{0}, \mathrm{U}, \tilde{1}\}$ and $\sigma=\{\tilde{0}, \mathrm{~V}, \tilde{1}\}$ be intuitionistic fuzzy topologies on $\mathrm{X}$ and $\mathrm{Y}$, respectively. Then the mapping $f:(X, \tau) \rightarrow(Y, \sigma)$ is defined by $f(a)=p, f(b)=q$, and $f(c)=r$. Then $f(\operatorname{gecl}(A)) \subseteq \operatorname{ecl}(f(A))$ holds for every intuitionistic fuzzy set $A$ of $X$, but $f$ is not intuitionistic fuzzy ge-continuous.

Theorem 5.11. A mapping $\mathrm{f}$ from an intuitionistic fuzzy $\mathrm{eT}_{1 / 2}$-space $(\mathrm{X}, \tau)$ to an intuitionistic fuzzy topological space $(Y, \sigma)$ is intuitionistic fuzzy continuous if and only if it is intuitionistic fuzzy ge-continuous.

Proof.

Necessity: Follows from remark (5.2).

Sufficiency: Let $A$ be an intuitionistic fuzzy closed set of $Y$, then $f^{-1}(A)$ is intuitionistic fuzzy ge-closed set in $X$, because $f$ is intuitionistic fuzzy ge-continuous, since $X$ is $e T_{1 / 2}$, so $^{-1}(A)$ is intuitionistic fuzzy closed in $X$. Hence $f$ is intuitionistic fuzzy continuous.

Remark 5.12. The composition of two intuitionistic fuzzy ge-continuous mappings may not be intuitionistic fuzzy ge-continuous. 
Example 5.13. Let $X=\{a, b\}, Y=\{p, q\}$ and $Z=\{s, t\}$ and intuitionistic fuzzy sets $U, V$, and $W$ are defined as follows

$\mathrm{U}=\{\langle\mathrm{a}, 0.5,0.5>,<\mathrm{b}, 0.7,0.3>\}, \mathrm{V}=\{<\mathrm{p}, 0.3,0.7>,<\mathrm{q}, 0.2,0.8>\}, \mathrm{W}=\{<\mathrm{s}, 0.6,0.4>,<\mathrm{t}, 0.4,0.6\rangle\}$.

Let $\tau=\{\tilde{0}, U, \tilde{1}\}, \sigma=\{\tilde{0}, V, \tilde{1}\}$, and $\eta=\{\tilde{0}, W, \tilde{1}\}$ be intuitionistic fuzzy topologies on $X, Y$, and $Z$, respectively. Then the mapping $f:(X, \tau) \rightarrow(Y, \sigma)$ be defined by $f(a)=p$ and $f(b)=q$ and the mapping $g:(Y, \sigma) \rightarrow(Z, \eta)$ be defined by $g(p)=s$ and $g(q)=t$. Then $f$ and $g$ are intuitionistic fuzzy ge-continuous but gof not intuitionistic fuzzy ge-continuous.

Theorem 5.14. If $\mathrm{f}:(\mathrm{X}, \tau) \rightarrow(\mathrm{Y}, \sigma)$ is intuitionistic fuzzy ge-continuous and $\mathrm{g}:(\mathrm{Y}, \sigma) \rightarrow(\mathrm{Z}, \eta)$ is intuitionistic fuzzy continuous, then, gof $:(X, \tau) \rightarrow(Z, \eta)$ is intuitionistic fuzzy ge-continuous.

Proof. If $A$ is intuitionistic fuzzy closed in $Z$, then $f^{-1}(A)$ is intuitionistic fuzzy closed in $Y$ because $g$ is intuitionistic fuzzy continuous. Therefore $(g \circ f)^{-1}(A)=f^{-1}\left(g^{-1}(A)\right)$ is intuitionistic fuzzy ge-closed in $X$. Hence gof is intuitionistic fuzzy ge-continuous.

Theorem 5.15. If $\mathrm{f}:(\mathrm{X}, \tau) \rightarrow(\mathrm{Y}, \sigma)$ and $\mathrm{g}:(\mathrm{Y}, \sigma) \rightarrow(\mathrm{Z}, \eta)$ are two intuitionistic fuzzy ge-continuous mappings and $(\mathrm{Y}, \sigma)$ is intuitionistic fuzzy $\mathrm{e}_{1 / 2}$-space, then gof $:(X, \tau) \rightarrow(Z, \eta)$ is intuitionistic fuzzy ge-continuous.

Proof. Let $A$ be intuitionistic fuzzy closed in $Z$, then $g^{-1}(A)$ is intuitionistic fuzzy ge-closed in $Y$ because $g$ is intuitionistic fuzzy continuous. Since $Y$ is $e^{T} T_{1 / 2}, g^{-1}(A)$ is intuitionistic fuzzy closed in $Y$, therefore (gof $)^{-1}(A)=f^{-1}\left(g^{-1}(A)\right)$ is intuitionistic fuzzy ge-closed in $X$, because $f$ is intuitionistic fuzzy continuous. Hence gof is intuitionistic fuzzy ge-continuous.

Theorem 5.16. Intuitionistic fuzzy ge-continuous image of an intuitionistic fuzzy GEO-compact space is intuitionistic fuzzy compact.

Proof. Let $f:(X, \tau) \rightarrow(Y, \sigma)$ be an intuitionistic fuzzy ge-continuous mapping from an intuitionistic fuzzy GEO-compact space $(X, \tau)$ onto an intuitionistic fuzzy topological space $(Y, \sigma)$. Let $\left\{A_{i}: i \in \Lambda\right\}$ be an intuitionistic fuzzy ge-open cover of $Y$, then $\left\{f^{-1}\left(A_{i}\right): i \in \Lambda\right\}$ is an intuitionistic fuzzy ge-open cover of $X$. Since $X$ is intuitionistic fuzzy GEO-compact, it has finite intuitionistic fuzzy subcover say $\left\{f^{-1}\left(A_{1}\right), \ldots, f^{-1}\left(A_{n}\right)\right\}$. Since $f$ is onto $\left\{A_{1}, \ldots, A_{n}\right\}$ is an intuitionistic fuzzy open cover of $Y$ and so $(Y, \sigma)$ is intuitionistic fuzzy compact.

Theorem 5.17. If $\mathrm{f}:(\mathrm{X}, \tau) \rightarrow(\mathrm{Y}, \sigma)$ is an intuitionistic fuzzy ge-continuous surjection and $\mathrm{X}$ is intuitionistic fuzzy GEO-connected, then $\mathrm{Y}$ is intuitionistic fuzzy $\mathrm{C}_{5}$-connected.

Proof. Suppose $Y$ is not intuitionistic fuzzy connected. Then there exists a proper intuitionistic fuzzy set $G$ of $Y$ which is both intuitionistic fuzzy open and intuitionistic fuzzy closed. Therefore $f^{-1}(G)$ is a proper intuitionistic fuzzy closed and intuitionistic fuzzy open set of $X$, because $f$ is intuitionistic fuzzy ge-continuous surjection. Hence $X$ is not intuitionistic fuzzy GEO-connected.

\section{References}

[1] K. T. Atanassov, Intuitionistic fuzzy sets, Fuzzy Sets and Systems, 20 (1986), 87-96. 2.1, 2

[2] K. Balchandran, P. Sundram, H. Maki, On generalized continuous map in topological spaces, Mem. Fac. Sci. Kochi Univ. Ser. A Math., 12 (1991), 5-13. 1

[3] V. Chandrasekar, D. Sobana, A. Vadivel, On fuzzy e-open sets, fuzzy e-continuity and fuzzy e-compactness in intuitionistic fuzzy topological spaces, Sahand Commun. Math. Anal., 12 (2018), 131-153. 2.10, 2.11, 2.12, 2.14, 2.15, 2.17, 2.18, $2.19,2.20$

[4] C. L. Chang, Fuzzy topological spaces, J. Math. Anal. Appl., 24 (1968), 182-190. 1

[5] D. Coker, An introduction to intuitionistic fuzzy topological space, Fuzzy Sets and Systems, 88 (1997), 81-89. 1, 2.2, $2.3,2.4$

[6] D. Coker, A. H. Es, On fuzzy compactness in intuitionistic fuzzy topological spaces, J. Fuzzy Math., 3 (1995), 899-909. 1 
[7] W. Dunham, N. Levine, Further results on generalized closed sets in topology, Kyungpook, Math. J., 20 (1980), 169-175. 1

[8] H. Gürcay, D. Coker, A. H. Es, On fuzzy continuity in intuitionistic fuzzy topological spaces, J. Fuzzy Math., 5 (1997), 365-378. 1, 2.14

[9] I. M. Hanafy, Intuitionistic fuzzy $\gamma$-continuity, Canad. Math. Bull., 52 (2009), 544-554. 2.14

[10] E. Hatir, A. Erdogan, Between closed sets and g-closed sets, Far East J. Math. Sci. (FJMS), 11 (2003), 289-301. 1

[11] Y. C. Kim, J. W. Park, Some properties of r-generalized fuzzy closed sets, Far East J. Math. Sci. (FJMS), 7 (2002), $253-268$. 1

[12] N. Levine, Generalized closed sets in topology, Rend. Circ. Mat. Palermo (2), 19 (1970), 89-96. 1

[13] F. G. Lupiáñez, Separation in intuitionistic fuzzy topological spaces, Int. J. Pure Appl. Math., 17 (2004), $29-34.1$

[14] F. G. Lupiáñez, Quasicoincidence for intuitionistic fuzzy points, Int. J. Math. Math. Sci., 2005 (2005), 1539-1542. 2.5

[15] F. G. Lupiáñez, Nets and filters in intuitionistic fuzzy topological spaces, Inform. Sci., 176 (2006), $2396-2404$.

[16] F. G. Lupiáñez, Covering properties in intuitionistic fuzzy topological spaces, Kybernetes, 36 (2007), 749-753. 1

[17] H. Maki, R. Devi, K. Balachandran, Generalized $\alpha$-closed sets in topology, Bull. Fukuoka Univ. Ed. Part III., 42 (1993), 13-21. 1

[18] H. Maki, R. Devi, K. Balachandran, Associated topologies of generalized $\alpha$-closed sets and $\alpha$-generalized closed sets, Mem. Fac. Sci. Kochi Univ. Ser. A Math., 15 (1994), 51-63. 1

[19] R. Malviya, On certain concepts in fuzzy topology, Ph.D. Dissertation, R. D. V. V. Jabalpur (M.P.), (1997). 1

[20] S. S. Thakur, J. P. Bajpai, Intuitionistic fuzzy sg-continuous mappings, Int. J. Appl. Math. Anal. Appl., 5 (2010), 45-51. 1

[21] S. S. Thakur, J. P. Bajpai, Intuitionistic fuzzy w-closed sets and intuitionistic fuzzy w-continuity, Int. J. Contem. Adv. Math., 1 (2010), 1-15.

[22] S. S. Thakur, J. P. Bajpai, Intuitionistic fuzzy rg $\alpha$-closed sets, International Journal of Fuzzy Systems and Rough Systems, 4 (2011), 67-73.

[23] S. S. Thakur, J. P. Bajpai, Intuitionistic fuzzy rw-closed set and intuitionistic fuzzy rw-continuity, Notes on Intuitionistic Fuzzy Sets, 17 (2011), 82-96.

[24] S. S. Thakur, J. P. Bajpai, On intuitionistic fuzzy gpr-closed sets, Fuzzy Inf. Eng., 4 (2012), 425-444. 1

[25] S. S. Thakur, R. Chaturvedi, Generalized continuity in intuitionistic fuzzy topological Spaces, Notes on Intuitionistic Fuzzy Sets, 12 (2006), 38-44. 1

[26] S. S. Thakur, R. Chaturvedi, Intuitionistic fuzzy rg-irresolute mappings, Varahmihir J. Math. Sci., 6 (2006), 199-204. 1

[27] S. S. Thakur, R. Chaturvedi, Regular generalized closed sets in intuitionistic fuzzy topological Spaces, Stud. Cercet. Stiint. Ser. Mat. Univ. Bacau, 16 (2006), 252-272.

[28] S. S. Thakur, R. Chaturvedi, Intuitionistic fuzzy rg-continuous mappings, J. Indian Acad. Math., 29 (2007), $467-473$. 1

[29] S. S. Thakur, R. Chaturvedi, Intuitionistic fuzzy gc-irresolute mappings, Math. Notae, 45 (2007/08), 59-65. 1

[30] S. S. Thakur, R. Chaturvedi, Generalized closed sets in intuitionistic fuzzy topology, J. Fuzzy Math, 16 (2008), $559-572$. $1,2.6,2.7,2.8,2.9,2.22$

[31] N. Turnali, D. Coker, Fuzzy connectedness in intuitionistic fuzzy topological spaces, Fuzzy Sets and Systems, 116 (2000), 369-375. 1

[32] S. S. Thakur, R. K. Khare, Fuzzy rg-closed mappings, Varahmihir J. Math. Sci., 3 (2003), 65-70. 1

[33] S. S. Thakur, R. K. Khare, Fuzzy regular generalized continuous mappings, J. Indian Acad. Math., 27 (2005), 355-380. 1

[34] S. S. Thakur, R. Malviya, Fuzzy gc-irresolute mappings, Proc. Math. Soc., 11 (1995), 184-186. 1

[35] S. S. Thakur, R. Malviya, Generalized closed sets in fuzzy topology, Math. Notae, 38 (1995), 137-140. 1, 2.21

[36] L. A. Zadeh, Fuzzy set, Information and Control, 8 (1965), 338-353. 1 